\title{
Maize transposable elements contribute to long non-coding RNAs that are regulatory hubs for abiotic stress response
}

\author{
Yuanda $\mathrm{Lv}^{1,2 \dagger}$, Fengqin $\mathrm{Hu}^{3 \dagger}$, Yongfeng Zhou ${ }^{2}$, Feilong $\mathrm{Wu}^{4}$ and Brandon S. Gaut ${ }^{2^{*}}$
}

\begin{abstract}
Background: Several studies have mined short-read RNA sequencing datasets to identify long non-coding RNAs (IncRNAs), and others have focused on the function of individual IncRNAs in abiotic stress response. However, our understanding of the complement, function and origin of IncRNAs - and especially transposon derived IncRNAs (TE-IncRNAs) - in response to abiotic stress is still in its infancy.

Results: We utilized a dataset of 127 RNA sequencing samples that included total RNA datasets and PacBio fl-cDNA data to discover IncRNAs in maize. Overall, we identified 23,309 candidate IncRNAs from polyA+ and total RNA samples, with a strong discovery bias within total RNA. The majority (65\%) of the 23,309 IncRNAs had sequence similarity to transposable elements (TEs). Most had similarity to long-terminal-repeat retrotransposons from the Copia and Gypsy superfamilies, reflecting a high proportion of these elements in the genome. However, DNA transposons were enriched for IncRNAs relative to their genomic representation by $\sim 2$-fold. By assessing the fraction of IncRNAs that respond to abiotic stresses like heat, cold, salt and drought, we identified 1077 differentially expressed IncRNA transcripts, including 509 TE-IncRNAs. In general, the expression of these IncRNAs was significantly correlated with their nearest gene. By inferring co-expression networks across our large dataset, we found that 39 IncRNAs are as major hubs in co-expression networks that respond to abiotic stress, and 18 appear to be derived from TEs.

Conclusions: Our results show that IncRNAs are enriched in total RNA samples, that most (65\%) are derived from TEs, that at least 1077 are differentially expressed during abiotic stress, and that 39 are hubs in co-expression networks, including a small number that are evolutionary conserved. These results suggest that IncRNAs, including TE-IncRNAs, may play key regulatory roles in moderating abiotic responses.
\end{abstract}

Keywords: Long non-coding RNA, Transposable elements, Abiotic stress, Co-expression network

\section{Background}

The functional component of any genome extends beyond its protein coding sequences. Much of the additional function is encoded by RNAs, which vary in size from small RNAs (sRNAs) of $<25$ nucleotides (nt) in length, to tRNAs of 70 to $\sim 90 \mathrm{nt}$ in length, to an even larger class of long non-coding RNAs (lncRNAs). lncRNAs are typically defined as being longer than 200 nt and containing no more than one short $(<100$ amino acids) open reading frame [1].

\footnotetext{
*Correspondence: bgaut@uci.edu

${ }^{\dagger}$ Yuanda Lv and Fengqin Hu contributed equally to this work.

${ }^{2}$ Department of Ecology and Evolutionary, Biology, UC Irvine, Irvine, CA, USA Full list of author information is available at the end of the article
}

lncRNAs represent a stunning proportion of transcriptional products. In mice, for example, an early study cataloged $\sim 34,000$ lncRNAs, representing one-third of all polyadenylated cDNAs [2]. More recent work has annotated $\sim 14,000$ lncRNAs in humans [3]. Work in plants has lagged somewhat behind, but plant lncRNAs have been identified based on various kinds of high throughput expression data. For example, microarrays have been used to detect 6480 lncRNAs from Arabidopsis thaliana [4]; single-stranded RNA sequence data have led to the identification of 2224 lncRNA transcripts in rice (Oryza sativa) [5]; and total RNAseq data have been employed to detect 7245 lncRNAs in maize (Zea mays ssp. mays) [6].

(c) The Author(s). 2019 Open Access This article is distributed under the terms of the Creative Commons Attribution 4.0 International License (http://creativecommons.org/licenses/by/4.0/), which permits unrestricted use, distribution, and 
At least three general properties of lncRNAs have become apparent from studies of both plants and animals. The first is that many lncRNAs are polyadenylated and capped, suggesting that they are transcribed and processed similarly to mRNAs [7]. However, lncRNAs can also be non-polyadenylated, and hence robust lncRNA discovery requires consideration of both polyadenylated and non-polyadenylated RNA samples. The second is that lncRNAs tend to be expressed at lower levels than coding genes, but with precise spatio-temporal patterns [3, 7-13]. A third general property is that some lncRNAs overlap with coding regions and sometimes contain parts of an exon; however, most originate from intergenic spaces (and these are sometimes called long intergenic RNAs or lincRNAs). Consistent with their origin from intergenic spaces, a large proportion of lncRNAs are either derived from transposable elements (TEs) or contain remnants of TEs. For example, Kapusta et al. [7] determined that $75 \%$ of human IncRNAs contained regions that appear to be derived from TEs.

Just as the origin and structures of lncRNAs are diverse, they play similarly varied functional roles. One major role is to act as templates for sRNA production, which in turn often contribute toward the epigenetic silencing of TEs $[14,15]$. Some lncRNAs perform other key functions, especially regulatory roles in cellular and developmental processes $[3,16]$. In plants, for example, lncRNAs have been shown to affect functions as diverse as phosphate signaling [17], flowering time [18], and susceptibility to pathogens [19]. Consistent with the hypothesis that IncRNAs play important regulatory roles, some lncRNAs are conserved among species and appear to be under purifying selection $[3,20,21]$.

A growing body of evidence also points to a potential role for plant lncRNAs in responses to abiotic and biotic stresses. A few studies have identified Arabidopsis lncRNAs that respond to salt, drought, heat and cold stresses, as well as phosphate starvation [22-24]. The expression of 28\% (1832 of 6480) of Arabidopsis lncRNAs was found to be significantly altered under biotic and/or abiotic stresses [4]. These findings - i.e., that lncRNAs are associated with stress responses - are particularly important in the context of crop species, because abiotic stresses affect crop yield and quality [13, 25-29]. However, the identification of lncRNAs during crop stress response remains largely unexplored, with a few notable exceptions. For example, 637 nitrogenresponsive lncRNAs and 664 drought-responsive lncRNAs have been identified in maize seedlings $[6,30]$. Similarly, 1010 and 1503 lncRNAs are known to be differentially expressed under abiotic stress in rice and in chickpea [31]. An important but challenging issue is to discover lncRNAs that are associated with abiotic stress responses and then to determine which lncRNAs function as key regulators, which serve as sRNA templates and which represent transcriptional noise.

Here we identify lncRNAs that relate to abiotic stress responses in maize. Our work extends previous maize lncRNA studies in at least three ways [6, 8, 30]. First, our efforts to detect lncRNAs are based on more expansive data. To perform lncRNA discovery, we have amassed 127 RNAseq datasets that were generated by different methods, in different tissues and across developmental stages, with a large subset generated in abiotic stress experiments, including salt, drought, heat, cold, UV and ozone stresses. The data include 89 RNAseq samples based on Illumina sequencing, 36 RNAseq datasets based on PacbioIsoSeq experiments, and two Illumina RNAseq datasets that were based on total RNA to potentially detect non-polyadenylated lncRNAs. Second, we investigate the relationship between $\mathrm{TEs}$ and lncRNAs. More than $85 \%$ of the maize genome consists of DNA derived from TEs [32], and we therefore expect that many lncRNAs exhibit sequence similarity to TEs. Thus far, however, the connection between lncRNA and specific TE superfamilies has not yet been investigated for maize. Third, we identify the subset of lncRNAs that are differentially expressed under abiotic stress to begin to narrow the set of candidates that function in stress response. To further narrow a candidate list of potentially functional lncRNAs, we also investigate co-expression of lncRNAs with neighboring genes and within expression networks [33, 34]. Bringing these diverse analyses together, we identify several lncRNAs that are hubs in co-expression networks that respond to abiotic stress and show that several of these hubs are lncRNAs derived from TEs.

\section{Results}

\section{Construction of transcripts and IncRNA discovery}

To discover lncRNAs and examine their expression during abiotic stress, we used 89 RNAseq samples, 2 total RNA-Seq samples and 36 Pacbio Iso-Seq samples. For the Illumina datasets, we extracted and cleaned $305 \mathrm{~Gb}$ of sequence data; on average $92.1 \%$ of Illumina reads per sample aligned successfully to the maize B73 v4 reference sequence [35]. Aligned reads from each Illumina sample were merged. We also collected and cleaned $\sim 1.98 \mathrm{~Gb}$ of IsoSeq sequences, aligned them to the B73 reference, and collapsed them for a total of 17,673 loci with 43,774 transcripts. We then combined the Illumina RNAseq and Pacific Biosciences (PacBio) IsoSeq data based on alignment of contigs to the reference, ultimately identifying a non-redundant set of 77,172 loci with 95,523 transcripts (Additional file 1: Fig. S1). Among these, 19,449 transcripts were found only in the total RNA sample. The set of 95,523 transcripts consisted of both 
coding transcripts and potential lncRNA transcripts. To identify the latter, we used a pipeline based on a combination of annotation programs and Pfam analyses (see Methods). Of the 95,523 assembled transcripts, CPC2 annotation identified 31,967 non-coding transcripts $(\mathrm{CPC} 2$ score $<-1)$, and 41,839 transcripts were deemed to be noncoding based on $\mathrm{CNCI}$ analysis. Of these two sets, 26,099 transcripts were longer than $200 \mathrm{bp}$ and were predicted to be non-coding by both $\mathrm{CPC} 2$ and $\mathrm{CNCI}$. These were further filtered by: i) comparing them to the Pfam database, retaining only those transcripts without a match (Blast, Evalue>1e-05) and ii) FPKM filtration, based on our requirement that FPKM had to exceed 1 in least one sample. The final dataset, which we consider high confidence lncRNAs, consisted of 23,309 transcripts (Table 1; Additional file 1: Fig. S1), representing 24\% of the total $(23,309 / 95,523)$. The average length of these candidate lncRNAs was $382 \mathrm{bp}$. None had an ORF $>100$ amino acids in length, as per our definition of lncRNAs (see Methods), but most (95.15\%) had one ORF. Among the 23,309 lncRNA candidates, 59.3\% (or 13,822 transcripts) were identified from polyadenylated (polyA+) RNAseq samples, and the remaining $40.7 \%$ (or 9487 transcripts) were from total RNA samples, representing potential polyA- transcripts (Table 1 ; hereafter we refer to IncRNAs from total RNAs as polyA- for simplicity). A file containing all the identified lncRNAs sequences, along with their genomic locations, is provided in DataS1.

The 23,309 lncRNAs were widely distributed across the 10 maize chromosomes (Additional file 1: Fig. S2). We also examined their location relative to annotated coding sequences within the maize genome. As expected from our search strategy, most lncRNAs $(87.9 \%, 20,499$ of 23 , 309) were intergenic, based on the output (a U class code) from gff compare. Only 185 lncRNAs were found to be intronic, with 29 and 156 of these as polyA- and polyA+ (Table 1). The few remaining high confidence lncRNAs corresponded to, or overlapped with, previously annotated lncRNAs in the B73 v4 reference (Table 1). Among the 20,499 lincRNAs, $44.7 \%$ (or 9153 of 20,499) were from total RNA datasets (i.e, potentially polyA-), representing a significant enrichment for lncRNAs within the total RNA samples (Pearson $X$-squared; $p<0.001$ ).
Most IncRNAs are derived from transposable elements Previous work has shown that a large fraction of IncRNAs are derived from TEs [7, 35], including maize lncRNAs [8]. These observations have led to the hypothesis that TEs contribute to the functional domains of lncRNAs [36]. However, previous papers have provided few details about the TE superfamilies that have contributed to lncRNAs or to the proportion length of individual lncRNAs that can be attributed to TEs. Accordingly, we examined our set of lncRNAs to identify which may be derived from a TE. To do so, we masked regions of our 23,309 high-confidence lncRNAs using a speciesspecific TE library (see Materials and Methods). Overall, we found that $65.69 \%$ lncRNAs $(15,312$ of 23,309$)$ overlapped with known maize TEs, which is a proportion similar to the previous maize study based on fewer lncRNAs [8]. Most (61\%, or 9341 of 15,312) TElncRNAs showed similarity to TEs over $\geq 90 \%$ of their length (Fig. 1a). Perhaps unsurprisingly, the proportion of polyA- IncRNAs that were masked by TE sequence was higher than that of polyA+ lncRNAs $(79.26 \%$ vs. $56.37 \%)$, which is a significant difference $(p<1 \mathrm{e}-5)$ (Fig. 1b). Hereafter we refer to lncRNAs with sequence similarity to TEs as "TE-lncRNAs".

We further investigated the superfamily of TEs that were similar to the 15,312 TE-lncRNAs. We found that $86 \%$ had sequence similarity to Long Terminal Repeat (LTR) retrotransposons of the Gypsy and Copia superfamilies (Table 2) and also that some of these TElncRNAs exceeded $3750 \mathrm{bp}$ in length (Fig. 1c). A much smaller proportion of TE-lncRNAs were derived from DNA transposons (Table 2); the longest of these were shorter than the longest TE-lncRNAs with similarity to Gypsy and Copia elements (Fig. 1c).

These observations raise an interesting question: Do LTR/Gypsy and LTR/Copia elements give rise to lncRNAs more often than expected, given their proportion of the genome? To address this question, we estimated the proportion length among all annotated TEs that were attributable to LTR/Gypsy, LTR/Copia and other element superfamilies, based on RepeatMasker analyses. We then compared these percentages to the proportion length among inferred TE-lncRNAs (Table 2). We found, for example, that LTR/Gypsy elements produced TE-IncRNAs at roughly the expected

Table 1 A summary of IncRNA discovery

\begin{tabular}{|c|c|c|c|c|c|c|}
\hline & Total & \%TE-IncRNA & lincRNA & intronic & $\begin{array}{l}\text { Fall within } \\
\text { annotated IncRNAs }\end{array}$ & $\begin{array}{l}\text { Overlap with } \\
\text { annotated IncRNAs }\end{array}$ \\
\hline polyA- & 9,487 & $79.26 \%$ & 9,153 & 29 & 189 & 116 \\
\hline polyA+ & 13,822 & $56.37 \%$ & 11,346 & 156 & 1,201 & 1,119 \\
\hline Total & 23,309 & $65.69 \%$ & 20,499 & 185 & 1,390 & 1,235 \\
\hline
\end{tabular}



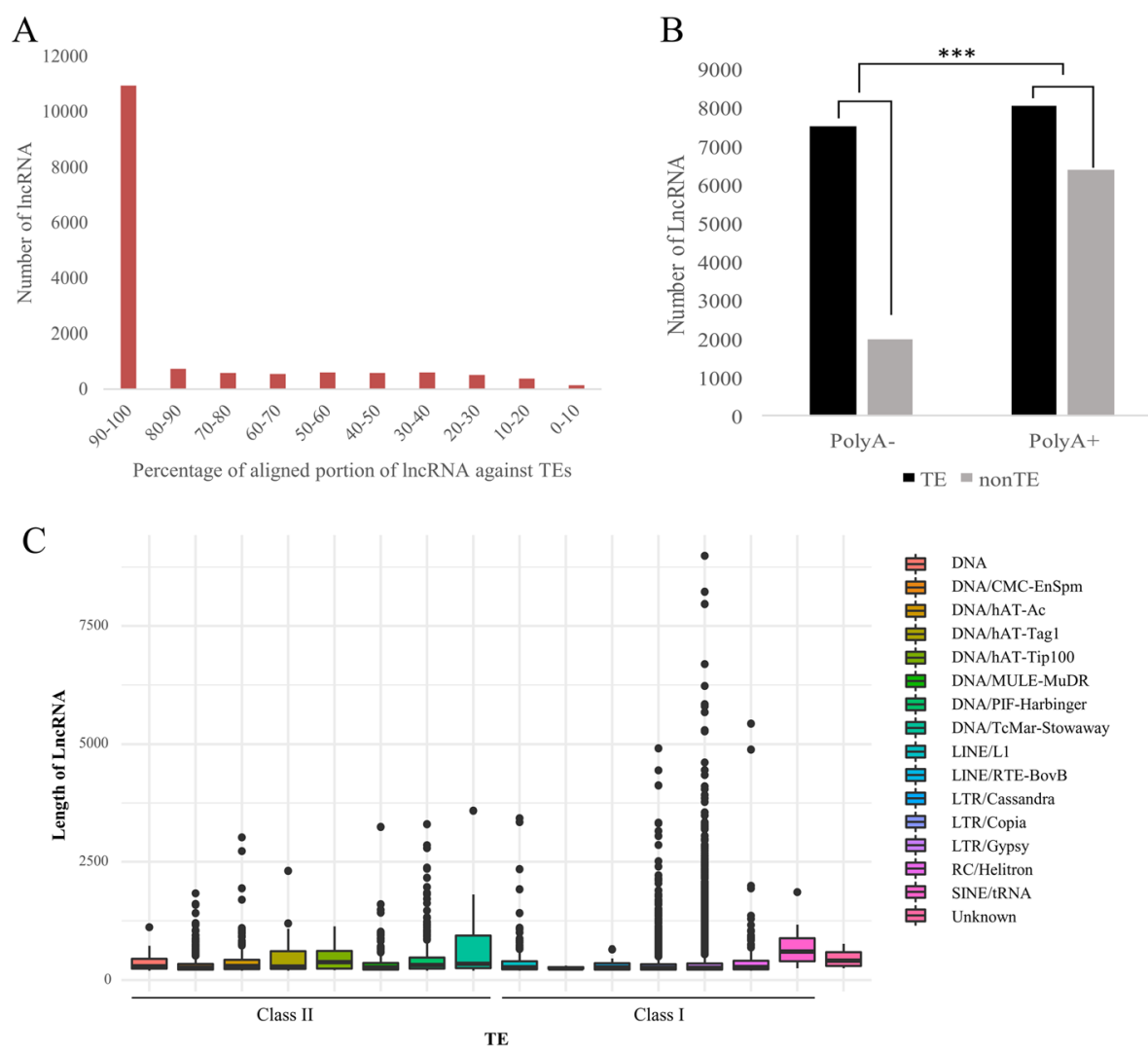

Fig. 1 The relationship between IncRNAs and TEs. a The histogram indicates the number of IncRNAs ( $y$-axis) relative to the percentage length ( $x$ axis) of IncRNAs that have similarity to TEs. $\mathbf{b}$ Numbers of IncRNAs that are polyA- (i.e., from total RNA) or polyA+ with similarity to TEs. The proportion of polyA- IncRNAs is significantly enriched for similarity to TEs. c The length distribution of TE-IncRNAs organized by their inferred TE superfamily of origin

proportion (61\% vs. 59\%), relative to their representation in the genome. However, LTR/Copia elements contributed TE-lncRNAs at a lower proportion than their proportion length among annotated TEs $(22 \%$ vs. $33 \%)$. Particularly notable is the fact that class II DNA elements produced TE-lncRNAs in our dataset at $\sim 2$ fold higher rate ( $12 \%$ vs. $6 \%$ ) than expected based on their total length among TEs in the genome (Table 2). Altogether, our results verify that the most maize lncRNAs derive from TEs, but they also indicated that different TE superfamilies give rise to TE-lncRNAs at different rates.

\section{Differential expression under abiotic stress}

One general feature of IncRNAs is that they are expressed at lower levels than protein coding genes, and they are often expressed tissue specifically $[3,6,8,23,37,38]$. We assessed the expression levels of coding and lncRNA transcripts based on their maximum FKPM across all of our 129 datasets and then averaged these maximum levels across transcripts. The results indicated that lncRNAs are expressed at lower levels than coding RNAs (Fig. 2a), with coding regions expressed at three-fold higher maximum levels, on average, than non-TE-lncRNAs (average FPKM: 12.57 vs. 4.30) and six-fold higher maximum levels, on average, than TE-lncRNAs (average FPKM: 12.57 vs. 2.04).

We next sought to identify coding genes and lncRNAs that were differentially expressed under abiotic stress. To do so, we contrasted a subset of our RNAseq samples that were generated from abiotic stress experiments that included both treatment and control RNAseq samples. For example, our samples included 12 RNAseq datasets that represent two control samples and two replicated treatment samples from each of four stresses (salt, drought, heat and cold) (Additional file 2: Table S1), all taken from V3 seedlings. Accordingly, we contrasted each stress treatment to the control, for a total of four contrasts (salt, drought, heat and cold) in V3 seedlings. Extending this approach to V4 and V6 seedlings across all the RNAseq data, we performed a total of 12 contrasts (Additional file 2: Table S1). These contrasts identified numerous differentially expressed coding genes and lncRNAs (Table 3). The various treatments identified $\sim 2000$ up- or down-regulated coding transcripts, on average, and a set of 1077 non-redundant lncRNAs that were either up- and down-regulated across treatments. 
Table 2 The proportion of base pairs attributable to different TE superfamilies based on the total length of inferred TE-IncRNAs and the $\mathrm{B} 73$ reference genome

\begin{tabular}{|c|c|c|c|}
\hline TE Class & Number of TE-IncRNAs & $\%$ total length of TE-IncRNAs & $\%$ total length of TEs in the genome \\
\hline \multicolumn{4}{|l|}{ Retroelements } \\
\hline SINE & 15 & $0.36 \%$ & $0.02 \%$ \\
\hline LINE/L1 & 151 & $1.28 \%$ & $0.96 \%$ \\
\hline LINE/RTE-BovB & 8 & $0.05 \%$ & $0.14 \%$ \\
\hline LTR/Cassandra & 47 & $0.23 \%$ & $0.10 \%$ \\
\hline LTR/Copia & 3917 & $22.45 \%$ & $32.39 \%$ \\
\hline LTR/Gypsy & 9566 & $61.53 \%$ & $59.64 \%$ \\
\hline Total & 13,704 & $85.90 \%$ & $93.25 \%$ \\
\hline \multicolumn{4}{|l|}{ DNA transposons } \\
\hline DNA & 28 & $0.28 \%$ & $0.03 \%$ \\
\hline DNA/CMC-EnSpm & 579 & $3.97 \%$ & $3.33 \%$ \\
\hline DNA/hAT-AC & 234 & $2.03 \%$ & $0.73 \%$ \\
\hline DNA/hAT-Tag1 & 20 & $0.56 \%$ & $0.08 \%$ \\
\hline DNA/hAT-Tip100 & 32 & $0.34 \%$ & $0.14 \%$ \\
\hline DNA/MULE-MUDR & 213 & $1.70 \%$ & $0.93 \%$ \\
\hline DNA/PIF-Harbinger & 291 & $2.80 \%$ & $0.64 \%$ \\
\hline DNA/TcMar-Stowaway & 35 & $0.41 \%$ & $0.08 \%$ \\
\hline Total & 1,432 & $12.10 \%$ & $5.96 \%$ \\
\hline Helitrons & 162 & $1.79 \%$ & $0.78 \%$ \\
\hline Unclassified: & 14 & $0.21 \%$ & $0.02 \%$ \\
\hline Total & 15,312 & & \\
\hline
\end{tabular}

Among the 1077 non-redundant lncRNA transcripts, many were differentially expressed in two or more treatments. For example, 679 lncRNAs were identified as differentially expressed across V3-V6 stages under heat treatment (Table 3; Fig. 2c; Additional file 4: Table S3, Additional file 1: Fig. S3). Of these, 29 lncRNAs were differentially expressed in all three developmental stages, but 79, 214 and 232 lncRNAs were specific to the V3, V4 and V6 stages, respectively. Interestingly, 40.50\% (32/ 79) heat-responsive lncRNAs at the V3 stage, $26.17 \%$ $(56 / 214)$ heat-responsive lncRNAs at V4 and $42.67 \%$ $(99 / 232)$ heat-responsive lncRNAs at V6 were also differentially expressed in response to other stress treatments, but not shared among developmental stages. These patterns implicate many lncRNAs as a common component of abiotic stress responses, but they also imply that these responses have temporal (i.e., developmental) specificity in leaves from V3 to V6 seedlings.

Interestingly, 529 non-redundant TE-lncRNAs were differentially expressed under one or more conditions. The proportion of differentially expressed TE-lncRNAs was lower than the proportion of all lncRNAs; TElncRNAs were $65 \%$ of the total proportion of lncRNAs, but constituted only 45 and $56 \%$ of up- and downregulated lncRNAs. Most of the differentially expressed
TE-lncRNAs had similarity to LTR/Gypsy and LTR/ Copia, as expected, but other TE families also contributed to differentially expressed TE-lncRNAs. For example, MSTRG.32907 exhibited similarities to LINE elements, MSTRG.73329 was similar to DNA/hAT-Ac elements, and MSTRG.37644 was an LTR/Gypsy elements. All of these were differentially expressed in leaves from V3 seedlings, but in different abiotic treatments (heat, cold and salt, respectively) (Fig. 4).

lncRNAs have been shown to be involved in cis regulation of neighboring genes. To investigate this possibility, we examined the correlation in expression between lncRNAs and their closest neighboring gene in either the $5^{\prime}$ or $3^{\prime}$ direction, yielding a dataset of 1077 differentially expressed lncRNAs and their neighboring genes. The lncRNAs were strongly $(r=0.48)$, and highly significantly $(p<2 \mathrm{e}-16)$ correlated with the expression of their closest neighboring gene (Fig. 2b), suggesting that lncRNAs may either be involved in cis regulation or are subject to some of the same cis regulatory features as their neighboring genes.

\section{Co-expression modules associated with stress responses}

Compared to coding genes and microRNAs, the function of lncRNAs in abiotic stress response remains largely 


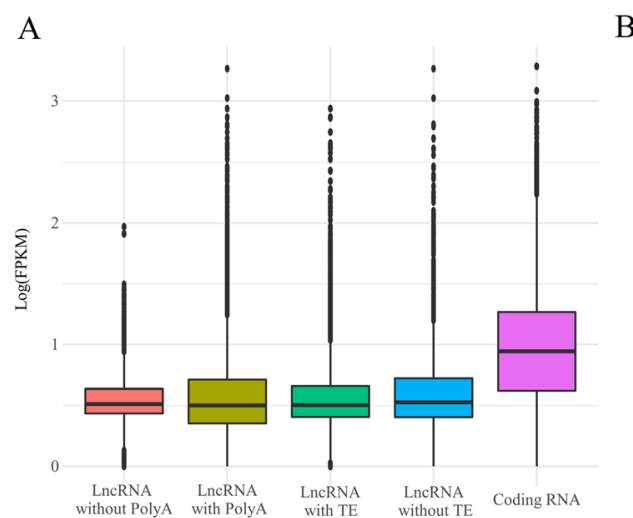

B

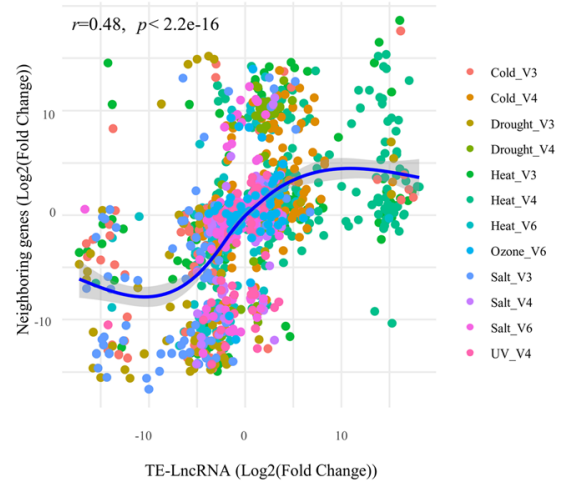

$\mathrm{C}$

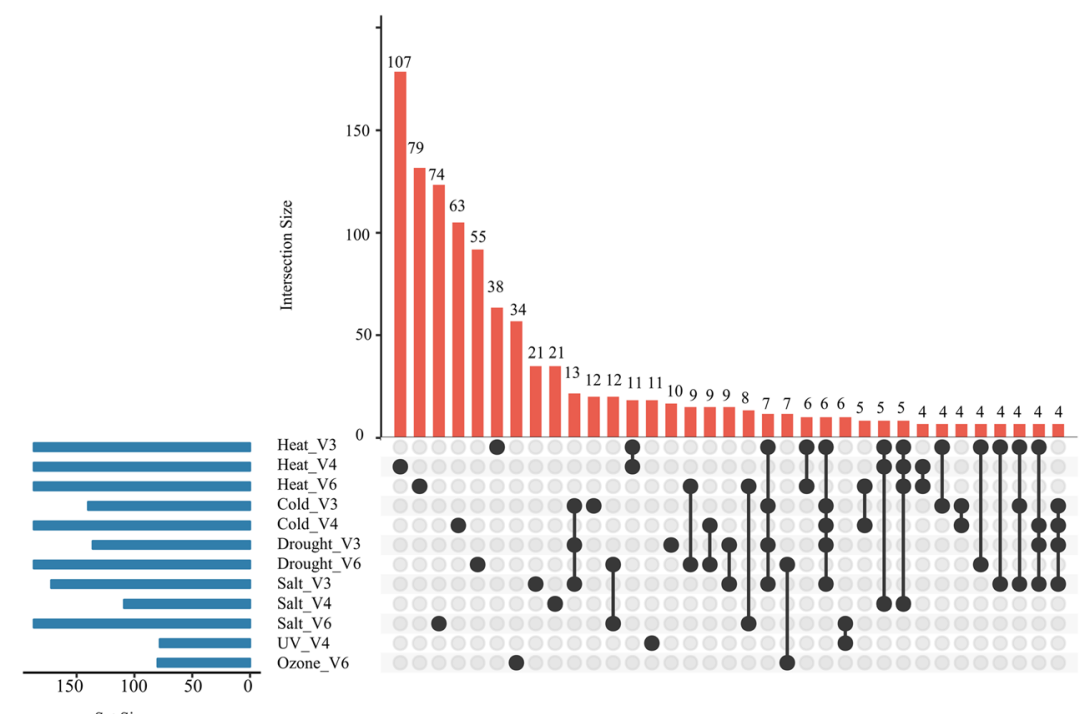

Fig. 2 Features of the expression dynamics of IncRNAs. a The Log2(FPKM) expression level of coding RNAs, IncRNAs and TE-IncRNAs, based on the maximum expression of each IncRNA across datasets. $\mathbf{b}$ The correlation (log2(Fold Change)) of TE -IncRNAs and the closest neighboring gene under different stress conditions. The blue curve indicates the best fit across all of the plotted points and clearly indicates a strong positive correlation from when log2(Fold Change) varies between roughly -5 and 5 . The linear correlation of $r=0.48$ is indicated in the graph. $\mathbf{c}$ This figure reports the number of differentially expressed IncRNAs under different stress conditions and developmental stages. The blue histogram on the left shows how many IncRNAs were differentially expressed under different conditions. The red histogram, coupled with the dot plot below, represent the distribution of differentially expressed IncRNAs among stress and stages. For example, the blue graph on the left indicates that 187 IncRNAs were differentially expressed under heat stress in V3. Of these, 38 were detected only under heat stress, as indicated by the graph in red, while 11 were differentially expressed under heat stress at both the V3 and V4 stages and 6 were differentially expressed under heat stress at both the V3 and V6 stages

Table 3 Numbers of differentially expressed genes, IncRNAs and TE-IncRNAs in maize seedlings under abiotic stress

\begin{tabular}{|c|c|c|c|c|c|c|c|c|c|c|c|c|}
\hline \multirow[t]{2}{*}{ Condition/Stage } & \multicolumn{3}{|l|}{ Heat } & \multicolumn{2}{|l|}{ Cold } & \multicolumn{2}{|c|}{ Drought } & \multicolumn{3}{|l|}{ Salt } & \multirow{2}{*}{$\begin{array}{l}\text { UV } \\
\text { V4 }\end{array}$} & \multirow{2}{*}{$\begin{array}{l}\text { Ozone } \\
\text { V6 }\end{array}$} \\
\hline & V3 & V4 & V6 & V3 & V4 & V3 & V6 & V3 & V4 & V6 & & \\
\hline Up-regulated & 2,051 & 3,279 & 2,892 & 1,273 & 3,161 & 1,260 & 2,560 & 1,292 & 2,348 & 3,114 & 2,752 & 1,712 \\
\hline Coding & 1,952 & 3,030 & 2,681 & 1,248 & 2,976 & 1,233 & 2,433 & 1,264 & 2,264 & 3,028 & 2,703 & 1,660 \\
\hline IncRNA & 84 & 223 & 181 & 23 & 159 & 24 & 112 & 22 & 64 & 74 & 36 & 45 \\
\hline TE-IncRNA & 36 & 92 & 86 & 6 & 64 & 8 & 59 & 5 & 21 & 31 & 13 & 19 \\
\hline Down-regulated & 1,511 & 3,411 & 3,407 & 1,450 & 2,420 & 1,543 & 1,904 & 1,266 & 2,944 & 3,183 & 2,740 & 738 \\
\hline Coding & 1,395 & 3,299 & 3,209 & 1,312 & 2,310 & 1,414 & 1,806 & 1,101 & 2,894 & 2,945 & 2,686 & 699 \\
\hline IncRNA & 103 & 97 & 101 & 117 & 93 & 112 & 80 & 150 & 45 & 208 & 42 & 35 \\
\hline TE-IncRNA & 66 & 38 & 118 & 70 & 39 & 63 & 38 & 101 & 15 & 133 & 14 & 15 \\
\hline
\end{tabular}


unknown. Computational construction of gene coexpression networks can be a valuable tool for linking lncRNAs and coding RNAs and also for beginning to infer potential biological functions, because co-expressed genes are often members of the same pathway or protein complexes, are often either functionally related, or are controlled by the same transcriptional regulatory program [33, 39-41].

We used the 89 Illumina RNA-seq datasets to build co-expression networks (see Methods and Table S1). WGCNA analyses identified 40 modules that comprise various nodes in the network. Of the 40 inferred modules, 16 were significantly correlated with stress treatments (Fig. 3, Additional file 1: Fig. S4, Additional file 4: Table S3, Additional file 5: S4). These 16 contained 7221 transcripts including 408 lncRNAs, of which 171 were TElncRNAs. Most of the 16 modules were associated with a single stress and developmental stage, but some were correlated with two or more stresses or stages (Fig. 3). For example, the ME_darkgreen module was highly correlated with drought at the V3 stage $\left(r^{2}=0.76, p<4 \mathrm{e}-18\right)$, but it was also significantly correlated with salt stress at the V3 $\left(r^{2}=0.21, p<0.05\right)$ and V4 $\left(r^{2}=0.29, p<0.005\right)$ stages. Similarly, the ME_salmon module correlated with drought $\left(r^{2}=0.25, p<0.02\right)$ and also salt stress at the V3 $\left(r^{2}=0.45, p<1 \mathrm{e}-05\right)$ and V4 stages $\left(r^{2}=0.38\right.$, $p<3 \mathrm{e}-04)$. Complete correlation information between modules and stress conditions and developmental stages are provided in Additional file 1: Fig. S4.

Recent work uncovered a temporal transcriptional logic underlying nitrogen (N) signaling in Arabidopsis [42]; we see similar logic based on developmental timing for abiotic stress responses. Consider the example of heat stress: the ME_tan module was correlated with V3 heat stress $\left(\mathrm{r}^{2}=0.89, p<4 \mathrm{e}-32\right)$, the ME_yellow module correlated with V4 heat stress $\left(\mathrm{r}^{2}=0.96, p<1 \mathrm{e}-49\right)$, and the ME_darkturquoise $\left(\mathrm{r}^{2}=0.43, p<2 \mathrm{e}-05\right)$ and $\mathrm{ME}_{-}$ pink $\left(\mathrm{r}^{2}=0.49, p<1 \mathrm{e}-06\right)$ modules were associated with heat stress in the V6 stage. These data suggest a developmental cascade of heat-responsive modules. To illustrate this graphically, we arranged the 16 associated modules by stress and development stage. Like heat stress, cold and drought stress were both associated with distinct modules at different developmental stages. There were exceptions, however, as both salt and UV stress associated with two modules in the V4 stage (Fig. 3).

Among the 16 significant modules, the most lncRNAs were associated with the ME_yellow module, which correlated with heat stress in the V4 stage $\left(\mathrm{r}^{2}=0.96, p<1 \mathrm{e}-49\right)$ and contained 147 lncRNAs and 65 TE-lncRNAs. Figure 4 details the expression pattern of this and other stress related modules. Given these modules, it is possible to extract the eigengenes from modules to infer function. For example, the eigengenes for the ME_yellow module were

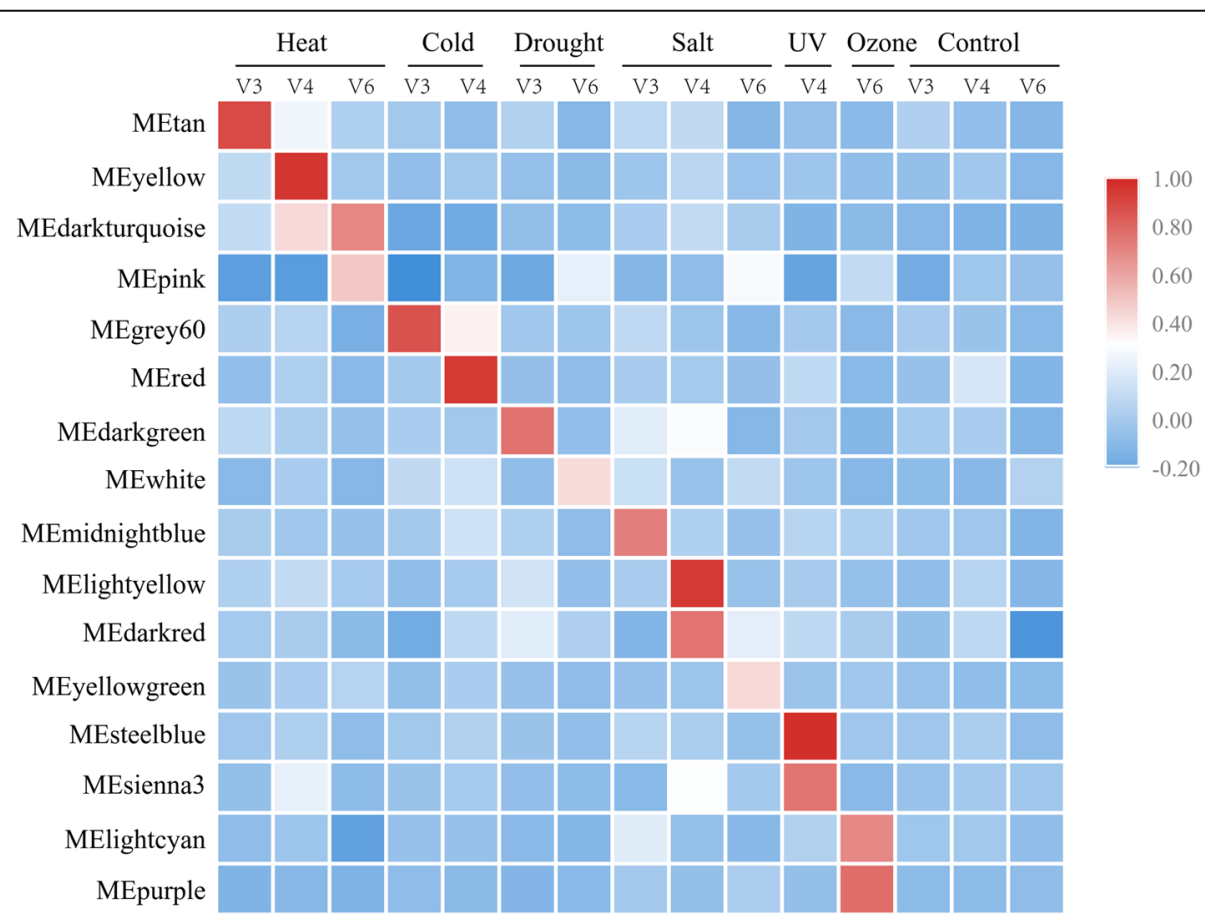

Fig. 3 A visual representation of the 16 modules that were significantly correlated with abiotic stress responses. All of the modules were associated with one stress condition and developmental stage, such that they exhibit a temporal cascade of stress responsiveness under different stresses and across V3 to V6 developmental stages. The scale of the heat map reflects the level of correlation ( $r$ ) among genes in an expression module for a specific abiotic stress (i.e., Heat, Cold, Drought, Salt, UV, Ozone) at a specific development stages (i.e., V3 to V6) 


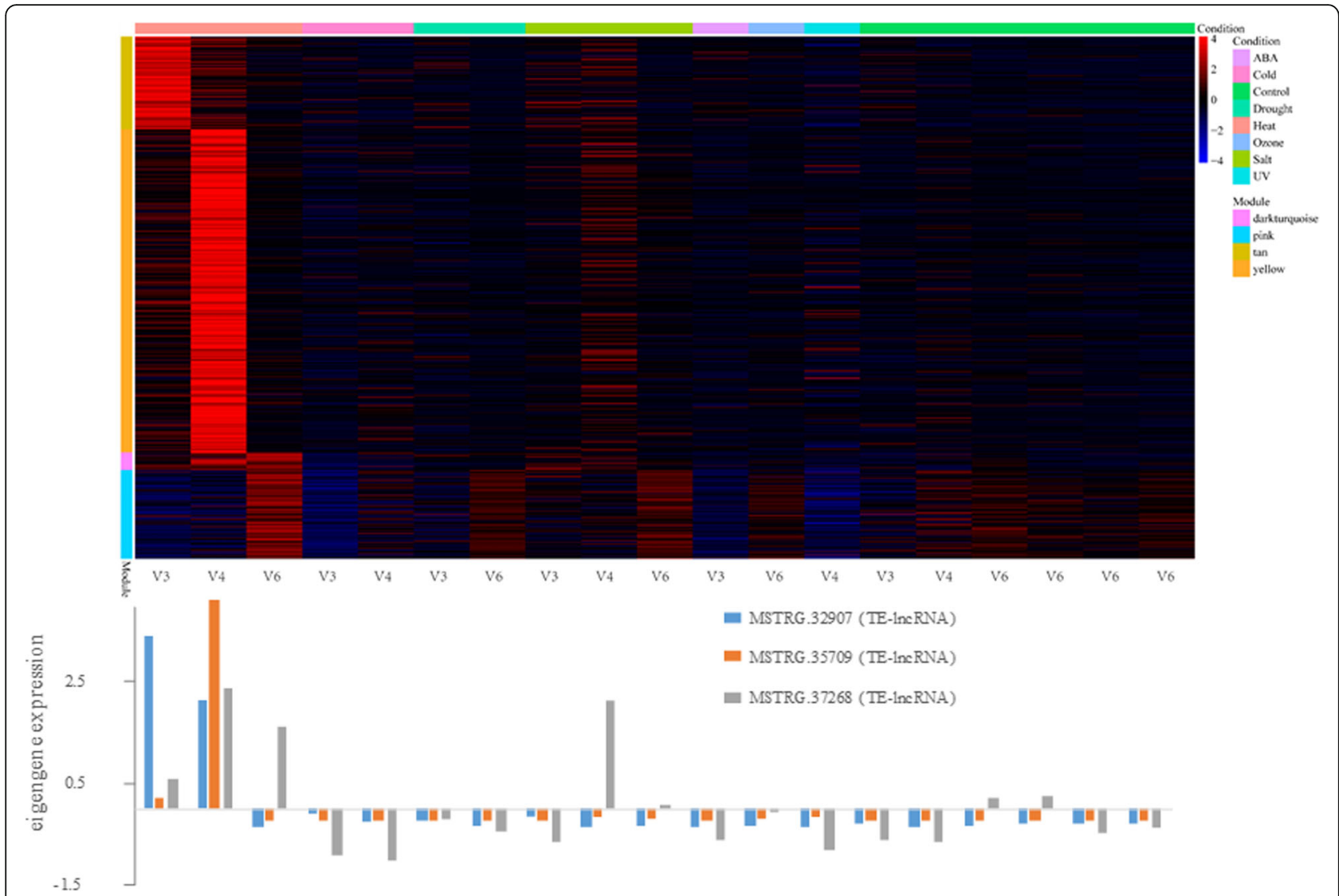

Fig. 4 IncRNA expression for four modules associated with heat stress. This figure consists of a heat map (top) and graphs of the expression of specific TE-IncRNAs (bottom) that were chosen because they were top three overrepresented IncRNAs in the four modules and had high interconnectivity. Top) The heat graph shows transcript expression levels for hub genes and IncRNAs in each module ( $y$-axis) and across conditions ( $x$-axis). The key to modules ( $y$-axis) and stress conditions ( $x$-axis) are shown on the right legend, with conditions also separated by developmental stage (bottom of $x$-axis). Warmer colors within the heat map indicate high expression, and cooler colors are low (or under) expression. This particular heat map illustrates that the four heat-associated modules are, as expected, highly expressed under heat stress, but not always at the same developmental stage. Bottom) The bar plots below the heat graph are eigen-IncRNA expression values selected from the top three overrepresented TE-IncRNAs with high interconnectivity. The $x$-axis is the same as the heat map, and the id of the TE-IncRNAs is provided by the color key. This graphs shows, again, that the TE-IncRNAs tend to be more highly expressed under heat stress, but with some dependence on developmental stage. Additional file 1: Figs. S5 to S9 present similar figures for modules associated with cold, drought, salt, UV and ozone stress, respectively

assigned into GO categories related to 'response to heat', 'response to high light intensity', 'heat acclimation, response to radiation', 'regulation of seedling development' and 'ER-nucleus signaling pathway'. The ME_darkturquoise $\left(\mathrm{r}^{2}=0.43, p<2 \mathrm{e}-05\right)$ and ME_pink $\left(\mathrm{r}^{2}=0.49, p<\right.$ 1e-06) modules were also associated with heat stress but in a later development stage (V6). These two modules contained 52 lncRNAs and 16 TE-lncRNAs, and their eigengenes exhibited significant enrichment of the GO terms 'intracellular ribonucleoprotein complex', 'HslUV protease complex', 'cytoplasmic translation' and 'intracellular membrane-bounded organelle' (Additional file 6: Table S5, Additional file 7: Table S6, Additional file 8: Table S7). Overall, GO-inferred functions helped verify that the modules reflect aspects of the stress response.

\section{LncRNAs are hubs in modules}

An interesting facet of the 16 stress-associated modules is that each contained both lncRNAs and TE-lncRNAs. We have mentioned that the ME_yellow module contained the most lncRNAs of the 16 modules, with 147 lncRNAs and 65 TE-lncRNAs, but other modules were similar in containing lncRNAs. For example, the ME_tan module, which is associated heat stress in V3, contained 26 lncRNAs and 9 TE-lncRNAs. An important question concerns the role of these lncRNAs in expression networks. One role, which is suggested by our results (Fig. 2b), is that some of the lncRNAs in modules are co-expressed with genes due to cis interactions. It is also possible, however, that lncRNAs regulate genes in trans. To investigate this possibility, we screened for key 'hubs', which we defined by 
high connectivity (i.e., intramodular connectivity within the top $10 \%$ of all members of the module), membership $>0.9$ and high significance $(p<0.01)$ in the module. Based on these filters, we identified 670 hubs that included 39 lncRNAs from different stress-responsive modules (Additional file 5: Table S4), of which 18 were TE-lncRNAs.

Considering the heat-responsive modules as an example, the 3 associated modules had 27 lncRNAs as hubs, out of 225 total lncRNAs, with 12 of the 27 categorized as TElncRNA. The 27 hub lncRNAs included transcript TElncRNAs such as MSTRG.32907 (TE-lncRNA, LINE/L1, $p$ <1.78E-04), MSTRG.35709(TE-IncRNA, LTR/Gypsy, $p<2.59 \mathrm{E}-114$ ), MSTRG.44074 (TE-lncRNA, DNA/hATAc, $p<2.11 \mathrm{E}-19$ ) and MSTRG.37268 (TE-IncRNA, DNA/ CMC-EnSpm, $p<1.63 \mathrm{E}-08)$. In Fig. 4 , we illustrate the expression patterns of three of the top-ranked hubs within the heat-stress associated modules, with the top-ranked hubs for the other five abiotic stresses in Additional file 1: Figs. S5-9. All of these hubs are expressed under stress and demonstrate high intramodular connectivity.

Many hubs in co-expression networks belong to transcription factors (TF) of families such as TCP, AP2/ EREBP, MYB, WRKY, NAC, bZIP [43-46]. We found interactions and potential crosstalk between lncRNAs and stress-responsive TFs from these families. In the heatresponsive modules, for example, hub lncRNAs such as MSTRG.32907, MSTRG.36825 and MSTRG.30107 and MSTRG.35709 were connected to TF families such as TCP, NAC, Dof and bHLH, which are known to respond to abiotic stress from previous studies (Fig. 5, 48-50].

These results suggest the possibility that lncRNAs - and more specifically, some TE-lncRNAs - act to regulate abiotic stress responses. If they play a functional role, one would expect them to be conserved over evolutionary time. We tested this idea by blasting each of the 39 hub lncRNAs to an evolutionary gradient of genomes that included sorghum, rice and Arabidopsis (Additional file 9: Table S8). Of the 39, 16 had strong hits $\left(\mathrm{e}<10^{-15}\right)$ to sorghum, a close relative to maize, and 4 of these 16 were TE-lncRNAs. Moreover, three of the hub lncRNAs had hits to rice, but zero TE-lncRNAs had rice hits, and none of the 39 hub lncRNAs had significant hits to Arabidopsis. Overall, these results suggest that $\sim 10 \%$ these lncRNAs have been conserved since the divergence of rice and maize, roughly 50 million years ago [47], and that $39 \%$ have been conserved since the divergence between sorghum and maize, roughly 16 million years ago [48].

\section{Testing the reliability of RNA-seq based inferences via qRT-PCR}

All of our inferences are based on bioinformatic analyses of RNAseq samples. To explore the reliability of these inferences, we performed a heat-stress experiment on maize B73 V3 seedings. The seedlings were subjected to $50{ }^{\circ} \mathrm{C}$ for $4 \mathrm{~h}$ (see Methods), and their RNA was extracted. We then subjected the samples to quantitative real-time PCR (qRT-PCR) to compare expression changes between replicated control and heat-treated seedlings. We focused on ten representative transcripts, including seven lncRNAs and three coding genes. Among this set of ten transcripts, six were significantly up-regulated under heat-stress and four were significantly down-regulated based on qRT-PCR (Fig. 6). We then compared experimental results to those based on RNAseq data, illustrating a high degree of consistency $\left(r=0.936 ; p<1 \times 10^{-4}\right)$ between inferences based on RNA-Seq and on qRTPCR (Fig. 6, Additional file 10: Table S9).

\section{Discussion}

In this study, we accumulated and mined an expansive dataset to identify lncRNAs in maize, particularly those that are expressed in response to abiotic stress. Bioinformatic analyses led to the identification of 23,309 lncRNAs, the largest collection yet identified from maize. We characterized these IncRNAs with respect to three features: $i$ ) their prevalence and origins, especially IncRNAs that appear to be derived from TEs, $i i$ ) their expression levels and patterns, including a detectable ciseffect, and iii) their potential for functioning in abiotic stress response, as inferred from the construction of coexpression networks.

\section{IncRNA identification and characterization}

By its very nature, lncRNA discovery is limited by a number of factors. It is first, of course, limited by the definition of lncRNAs that have been used in the literature - i.e., an RNA molecule $>200$ bp with at most one ORF or overlapping exon of $<100$ codons [1]. Following precedence, we have adopted this definition for lncRNA discovery, but it bears remembering that some of these could in fact be translated because they contain short ORFs. A second limitation is the fact that our search strategy did not include lncRNAs that overlapped with (or contained) an annotated exon. We applied this limitation purposefully, to avoid mis-classification based on fragmented RNA molecules or contigs. For that reason, however, our work likely underrepresents lncRNAs derived from genes and so some of our estimates may be inaccurate. For example, if many lncRNAs are derived from genic regions, then our estimate of the proportion of lncRNAs that are derived from TE-lncRNAs is an overestimate. It is worth noting, however, that our estimate of the proportion of TE-lncRNAs (65\%) is similar to a previous, smaller maize study that estimated $68 \%$ of lncRNAs were derived from TEs [8]. A third limitation is that the completeness of lncRNA discovery relies critically on the number of tissue and developmental samples that are available. With the exception 


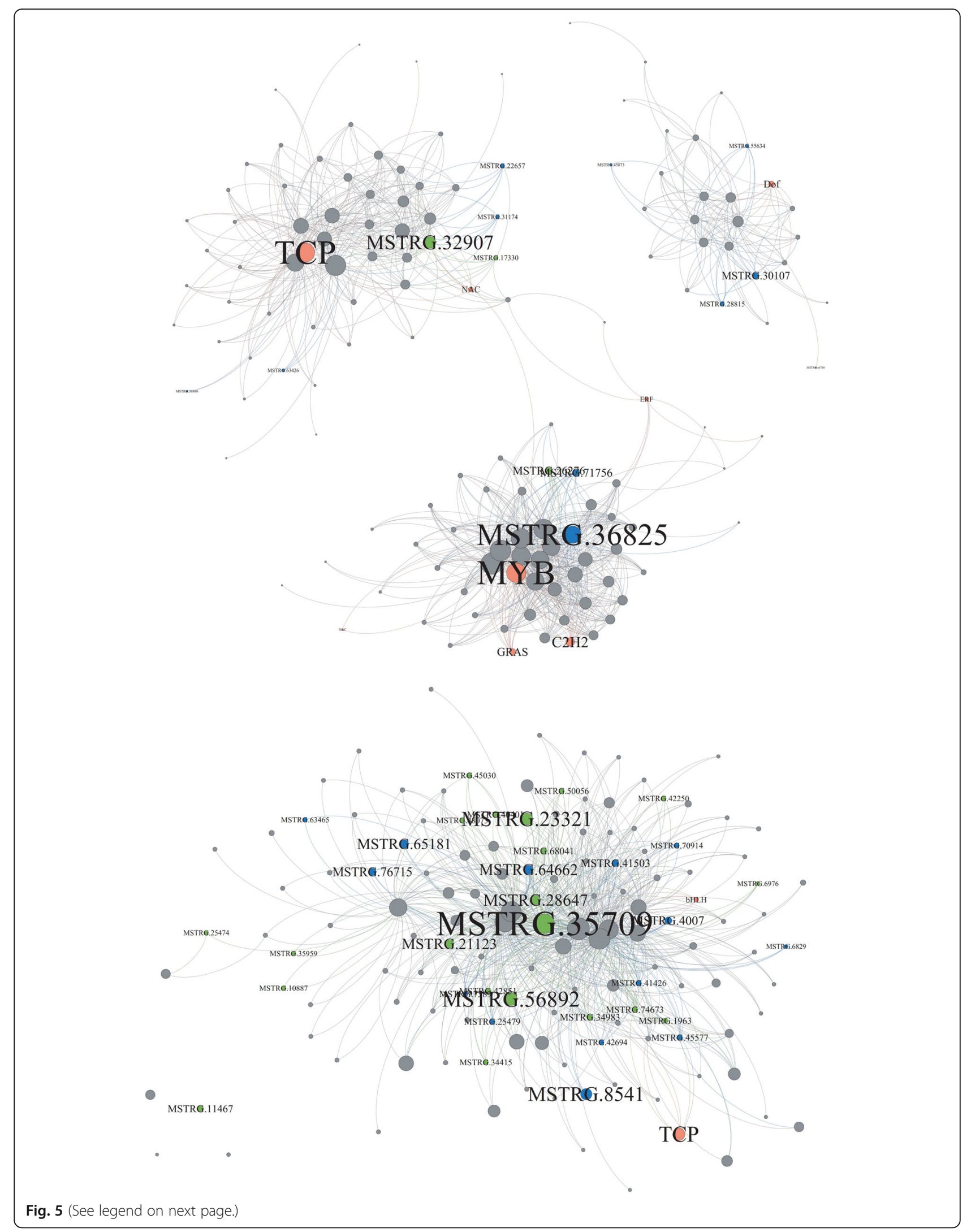


(See figure on previous page.)

Fig. 5 The networks of four heat-responsive modules. The four modules are, the ME tan module (top left), the ME yellow module (top right), the ME_pink module (middle), and the ME_darkturquoise module (bottom). In each network diagram, the green circles represent TE-IncRNAs; the blue color represents nonTE-IncRNAs; the orange dots represent known transcription factors from various families (e.g., TCP), and grey circles represent coding RNAs. The size of the dot represents intramodular connectivity, with larger sizes representing higher connectivity. From these networks, we can infer that IncRNAs and TE-IncRNAs are sometimes as or more interconnected than transcription factors

of $A$. thaliana, for which lncRNA discovery was based on 2000 microarray transcriptomes, most plant studies have been limited to only a handful of samples, suggesting that there is still much to learn about the lncRNA complement within and among plant species. Although our study focuses on only one tissue (i.e., leaves from seedlings of different developmental stages), it greatly expands lncRNA discovery in maize because previously the most RNAseq samples used for lncRNA discovery was 30 [8].

Our RNA datasets were highly enriched for polyadenylated (polyA+) transcripts, because it consisted of 36 PacBio fl-cDNA datasets, 89 RNAseq datasets and only two total RNA datasets. Nonetheless, fully $44 \%$ of intergenic lncRNAs were identified from the total RNA data, representing a disproportionately large number relative to polyA+ data. This observation superficially suggests that far more lncRNAs are polyA-, which is an important point to consider when one considers that most - but not all $[6,49,50]$ - IncRNA surveys in plants have relied solely on RNAseq samples and not total RNA samples. Previous work has also suggested that the ratio of polyA- and polyA+ IncRNAs may be a function of growth conditions and external stresses [13]. A fuller understanding of lncRNAs may require more substantial investments in total RNA datasets.

\section{Most IncRNAs are TE-IncRNAs}

Given our identification of 23,309 lncRNAs, we next sought to characterize their loci of origin and particularly to identify those that likely originated from TEs. We found that $\sim 65 \%(15,312)$ of IncRNAs contained similarity to known TEs. Of these, most (61\%, 9341 of 15,312 ) were similar to TEs over $>90 \%$ of their length, suggesting they were derived solely from TEs. As we noted above, our estimates of the proportion of TElncRNAs could be too high, based on our search strategy. However, it is also not surprising that we identified

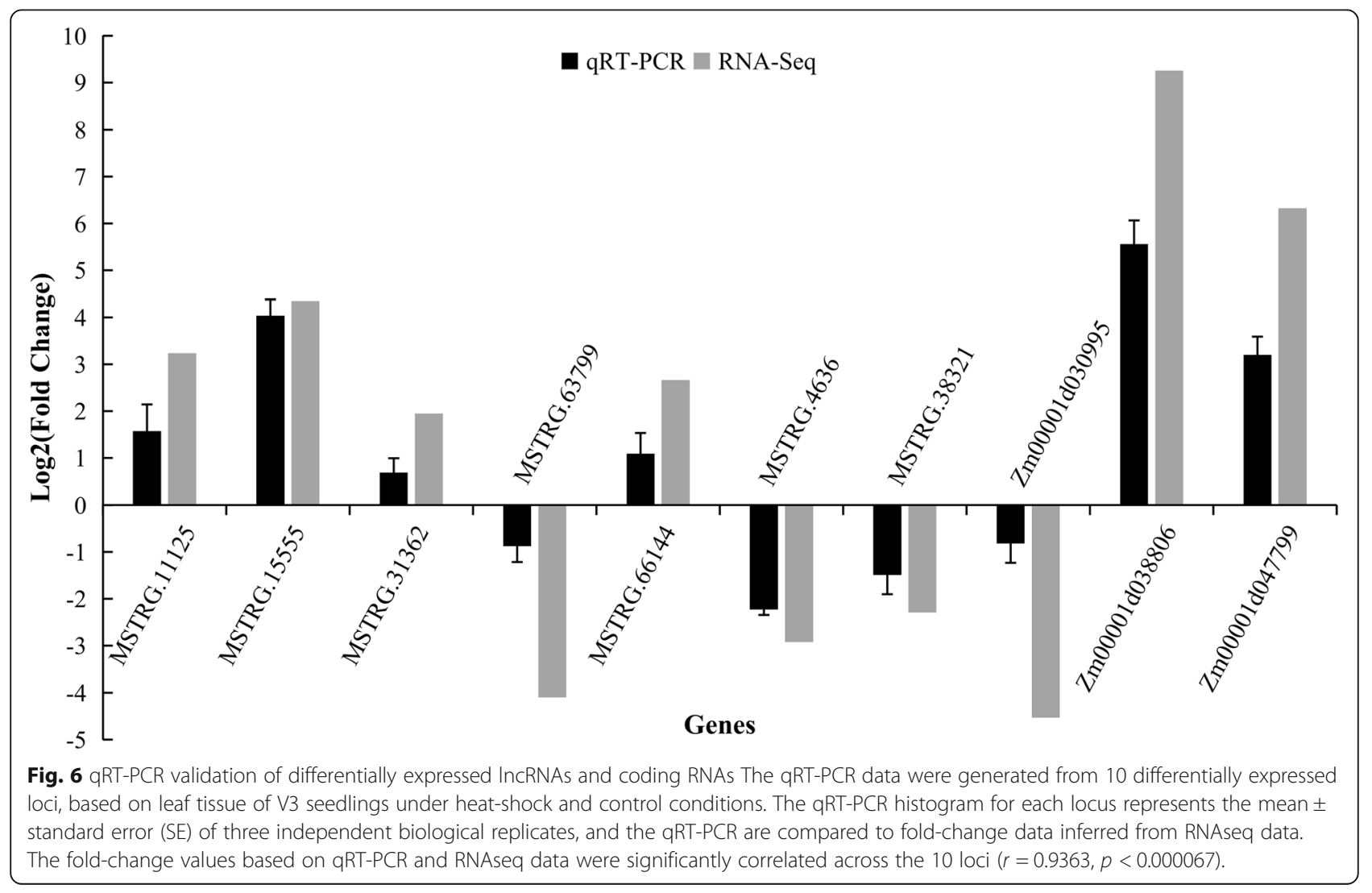


a high proportion of TE-lncRNAs, for at least three reasons. First, previous studies in mammals have demonstrated that most lincRNAs derive from TEs [7, 35]. Second, the maize genome is replete with TEs, with $>85 \%$ of the genome estimated to consist of DNA derived from TEs [32]. Finally, an important function of lncRNAs is to be precursors for small RNAs, which in turn contribute to TE silencing via sequence homology $[8,51-53]$.

We also investigated the TE families from which TElncRNAs originated. Most of the TE-lncRNAs were derived from LTR/Gypsy and LTR/Copia families (Table 2), reflecting their preponderance in the maize genome $[32,53]$. IncRNAs derived from LTR/Gypsy elements were represented in a similar proportion to their genomic proportion (by length) among the TEs we investigated in our study (Table 2). However, LTR/Copia elements were underrepresented in the TE-lncRNA dataset relative to their combined lengths in the genome, $22 \%$ versus $33 \%$. This suggests that LTR/Copia elements do not produce lncRNAs as readily as LTR/Gypsy elements, at least within our data. The reasons for the difference between LTR/ Copia and LTR/Gypsy are presently unclear, but one can consider two broad categories: TE age and TE location. For the former, older elements might be expected to be in a deeply-silenced epigenetic state that relies primarily on the maintenance of methylation during cell division rather than an active epigenetic response that enlists lncRNAs [54]. For the latter, one might expect LTR/Copia elements to be in genomic locations that are transcribed. In fact, however, the opposite is true, because LTR/Gypsy elements tend to be concentrated in pericentromeric regions [32] where there may be less active transcription and less ongoing silencing. In contrast, LTR/Copia elements tend to accumulate preferentially in euchromatic regions [32] that tend to be more transcriptionally active. Class II DNA elements also tend to be located near genes and euchromatic regions, but unlike LTR/Copia elements they produce lncRNAs at about a 2 -fold higher than implied by their genomic lengths (Table 2). To sum: we have shown that TE superfamilies over- and under-produce lncRNAs relative to their genomic representation based on our extensive collection of datasets, but the ultimate causes of these differences remain unclear.

\section{Levels and patterns of IncRNA expression}

Several previous papers from both plants and animals have shown that lncRNAs tend to be expressed at lower levels than bona fide genes and that they also tend to show tissue-specific patterns of expression [3, 7-12]. We have verified the former by recording the maximum FPKM for each lncRNA transcript across datasets; on average, IncRNAs are expressed at 4-fold lower levels than genic transcripts by this metric (Fig. 2a). Unfortunately, we cannot verify that lncRNAs have more tissue specific expression than genes, because the bulk of our data were isolated from leaves. We can, however, verify that they have lower entropy than genes, on average (Average Shannon Entropy $=2.10$ for coding genes vs. 1.13 for lncRNAS), because the lncRNAs consistently lack expression evidence under more conditions.

Of the 13,822 polyA+ IncRNAs, we found that 1077 (7.79\%) were differentially expressed under stress conditions, including 529 TE-lncRNAs. These TE-lncRNAs provided an opportunity to assess whether they could be linked to the expression of nearby genes, indicating some sort of cis-regulatory pattern, as has been observed in other species $[20,55,56]$. TE-lncRNAs were significantly correlated $\left(\mathrm{r}^{2}=0.48 ; p<2.0 \mathrm{e}-16\right)$ with their nearest neighboring genes (Fig. 2b), suggesting that TE-lncRNAs may either be involved in cis regulation or are subject to some of the same cis regulatory features as their neighboring genes, such as open chromatin structure.

\section{IncRNAs, abiotic stress and coexpression modules}

This study was designed specifically to identify stressresponsive lncRNAs. We approached this problem in two ways. We first identified differentially regulated lncRNAs from a series of controlled experiments for heat, cold, drought and salt stress. These experiments were based on leaf tissue from seedlings of the V3, V4 and V6 stages. Comparing the stress treatment to their corresponding control at the appropriate developmental stage across 12 different contrasts, we identified 1077 lncRNAs with evidence for differential expression. This observation corroborates previous studies in suggesting that IncRNAs may be differentially regulated under stress [6, 22-24, 30,31], but it provides no indication whether the differentially regulated lncRNAs are a byproduct of stress responses or play a functional role. There is, however, a large gap between observing differential expression and proving function. As a first step toward bridging this gap, we have built co-expression networks based on both coding RNAs and lncRNAs from 89 RNAseq datasets, yielding a total of 40 coexpression modules. Of these, 16 were significantly associated with stress responses, and GO annotations of these modules were generally consistent with their inferred response functions. One interesting facet of these 16 modules is that they demonstrate clear patterns across developmental time (Fig. 3), suggesting that temporal hierarchies are important for plant responses to environmental stress.

It is difficult to infer function from co-expression modules [57], but studies have shown that genes with high connectedness tend to be functionally essential $[58,59]$. We were therefore particularly interested whether any of our lncRNAs are included within co-expression networks and particularly whether they are 'hubs' within network 
modules. Of the 16 modules that were significantly associated with stress responses, we identified 670 hubs, many of which corresponded to genes from known transcription factor families (Fig. 5). Of these 670 hubs, 39 were lncRNA transcripts. These represent our best candidates for lncRNAs that function in stress response, potentially as trans-acting regulatory factors. Consistent with this last conjecture, several of these lncRNA hubs were connected to genes from known TF factors [60-62]. Moreover, $10 \%$ of these lncRNAs yielded strong blast hits to rice, suggesting some measure of evolutionary conservation consistent with functional constraint, at least for this subset.

One somewhat surprising finding is that 18 of the 39 IncRNA hubs are related in sequence to - and perhaps derived from - TEs. This observation raises the intriguing idea that TE exaptation can occur at the level of lncRNAs. It is now well known that TE exaptation contributes to many aspects of genome function, including protein coding genes and especially functional regulatory elements [63-65]. The location of TE-lncRNAs as hubs, along with their connectedness to known TFs, suggests that a small subset of TE-derived lncRNAs may function as trans-acting regulatory factors in maize. If true, these hubs appear to have been recruited recently, given that only four of 16 yield strong hits to the sorghum genome. Clearly additional work is required to prove that these TE-lncRNAs function as hypothesized in abiotic response, but their centrality in co-expression modules is nonetheless an intriguing result that is consistent with previous findings showing that most IncRNAs are derived from TEs [7] and that IncRNAs can play central regulatory roles in plant and animal development [63].

\section{Methods}

\section{Sample collection}

In this study, we gathered 36 Pacbio Isoseq datasets that were sampled from different tissues [66] and 91 illumina RNAseq datasets that were sampled from leaves of maize B73 [6, 67-69] (Additional file 2: Table S1, Additional file 1: Fig. S1). Of the Illumina datasets, 89 represented polyA+ transcripts and two were based on total RNA, which includes putative polyA- transcripts. The datasets were used for three purposes: IncRNA discovery, differential gene expression analyses, and the inference of gene co-expression networks. All of the 129 datasets were used for lncRNA discovery. A subset of 71 of the 91 RNAseq datasets were employed for differential gene expression analyses (Additional file 2: Table S1); these included replicated control and treatment samples from experiments that tested the effects of drought, salt, heat, cold, UV and ozone treatments on gene expression. Finally, all of the 89 polyA+ Illumina RNAseq datasets were used for inferring gene coexpression networks. The 89 Illumina datasets represented a developmental series sampled from leaves of $\mathrm{V} 3, \mathrm{~V} 4$ and V6 seedlings; we take advantage of this developmental series in some network analyses (Additional file 2: Table S1, Additional file 1: Fig. S1).

\section{Data processing and alignment}

Raw data were converted into the FASTQ-formatted file by the Fastq-dump program from the SRA Toolkit (https:/github.com/ncbi/sratoolkit). For Illumina data, the SolexaQA++ v3.1 program [70] was employed for quality trimming, using the Q20 value. After trimming, any reads $<50 \mathrm{bp}$ were removed. Cleaned reads were then aligned to the B73 reference genome sequence (v4, http://plants.ensembl.org) using the STAR aligner program [71] with default parameters. Aligned reads were assembled into transcripts by the StringTie program, using the RABT (reference annotation-based transcript) assembly algorithm [72]. For the Pacbio IsoSeq data, reads were aligned to the $\mathrm{B} 73$ reference genome using the Minimap2 program [73]. Unique isoforms were collapsed, based on genome alignment by Cupcake ToFU (https://github.com/Magdoll/cDNA_Cupcake). Subsequently, the assembled transcripts from Illumina RNAseq and Pacbio IsoSeq were merged using StringTie, which yielded a non-redundant unified set of transcripts.

\section{Computational identification of intergenic and intronic IncRNAs}

To find lncRNAs, a strict computational strategy was performed as described by Lv et al. (2016) that and consisted of four steps. First, non-redundant transcripts were submitted to annotation programs to evaluate their coding potential. We used two annotation programs CPC2 [74] and CNCI [75] - and focused on transcripts that were identified as having no coding potential by both programs as candidate lncRNAs. Second, we submitted candidates to the Pfam database using Pfam_scan script (ftp://ftp.ebi.ac.uk/pub/databases/Pfam/), which aligns transcripts with HMMER [76]. We filtered any transcripts that aligned to known protein families at an Evalue $<1 \mathrm{e}-05$. Third, we compared the remaining transcripts to reference annotations using gffcompare [77], which outputs various codes to designate the relationship of transcripts to annotated coding regions. We retained transcripts with class codes " $i$ ", which indicates that a transcript is fully contained within a reference intron, and "u", which designates transcripts that are not obviously related to known coding regions, for further analyses. This last step is likely to miss some sense and anti-sense lncRNAs that derive from coding regions but also limit false positives based on incompletely assembled coding transcripts. Finally, we retained transcripts as high confidence lncRNAs if they passed all of the previous four steps, if they were longer than $200 \mathrm{bp}$, and if 
they had an FPKM (fragments per kilobase of exon model per million reads mapped) $>1$ in at least one of our sample datasets. To determine the relationship of high-confidence lncRNAs to TEs, we masked the lncRNA sequences to identify TE domains. Masking was based on the maize-specific library of Repbase database (www.girinst.org) and was performed by RepeatMasker (www.repeatmasker.org).

\section{Gene expression analyses}

We performed two separate types of analyses based on gene and lncRNA expression data. The first analysis was differential expression analysis based on comparisons between stress and control data (Table S1). To perform these analyses, high quality reads were aligned to the B73 reference using the STAR program [71]. For reads that mapped to multiple locations, we removed alignment reads with a mapping quality $<20$, based on SAMTools [78]. Raw counts were quantified using the featureCounts program [79], and the FPKM value per gene was calculated using a custom Perl script. The DESeq2 package [80] was used to perform pairwise comparisons between samples to identify differentially expressed transcripts. To identify differentially expressed genes (DEG), we relied on two criteria: the Log2(fold change) had to be $>1$ and the adjusted $p$-value from DEseq analyses had to be $p$-adj $<0.05$.

The second type of analysis was the inference of coexpression networks. To construct networks, expression profiles were extracted from each gene and lncRNA, and expression levels were normalized using variance stabilizing transformation in DESeq2 [80]. Co-expression correlations among lncRNAs and genes were based on Pearson correlations with $\mathrm{R}^{2} \geq 0.8$ across the 89 RNAseq datasets. An unsigned co-expression network was inferred using the WGCNA package [81] with an optimal soft threshold $=12$. Modules within the network were assigned using Topological Overlap Matrix (TOM). The correlations between modules and stress treatments were calculated and plotted, and then the significant stress-responsive modules were extracted for further analysis. Co-expressed networks were visualized by the Gephi program [82].

\section{Gene ontology enrichment analysis}

The eigengene probes of each stress-responsive module were assigned putative functions by searching against the UniProt protein database [83]. Searching was based on using the Blastx program [84], using a cut-off evalue $\leq 1 \mathrm{e}-10$. Coding eigengenes were then submitted to the AgriGO v2 online toolkit [85] for gene ontology term enrichment. A Fisher's exact test was applied for the enrichment analysis and the $p$ value was adjusted using the Bonferroni method, with an experiment-wide significance level of 0.05 .

\section{Experimental stress treatment, RNA extraction and qRT- PCR analysis}

The maize inbred line B73 was germinated in a greenhouse at JAAS (Jiangsu Academy of Agricultural Sciences). Seedlings at the three-leaf (V3) stage were then incubated at $50^{\circ} \mathrm{C}$ for $4 \mathrm{~h}$ for heat stress treatment, as described by Makarevitch et al. [69]. Control plants were retained under a temperature of $25^{\circ} \mathrm{C}$. Leaves from three independent biological replicates were collected and processed for RNA extraction and first strand cDNA synthesis according to PrimeScriptTM ${ }^{\mathrm{RT}}$ Master Mix (TaKaRa). qRT-PCR was performed using SYBR Premix DimerEraser $^{\mathrm{m}}$ kits (Takara) on a Real Time PCR System (Roche LightCyclerR 96, USA), according to the manufacturer's instructions. Quantification results of target transcripts were calculated using the comparative 2- $\Delta \Delta \mathrm{CT}$ method. Primers were designed using Primer Primer5 [86] and can be found in Additional file 10: Table S9.

\section{Supplementary information}

Supplementary information accompanies this paper at https://doi.org/10. 1186/s12864-019-6245-5.

\begin{abstract}
Additional file 1: Figure S1. A schematic showing the data used in this paper, the bioinformatic pipeline for IncRNA identification, numbers of genes and identified ICRNAs, and some features of downstream IncRNA analyses. Figure S2. The chromosomal distribution of IncRNAs. Density was plotted across each chromosome. Figure S3. Differential expressed genes (DEGs) under different abiotic stresses. A-D, Venn diagram of DEGs under different stresses across different development stage V3-V6. E-G, Venn diagram of DEGs under different stress conditions at one stage. Figure S4. The correlation values ( $r$ ) between each of the inferred coexpression modules and the specific traits (e.g., Heat, Cold) at different development times (e.g., V3 to V6). A subset of these data for the top 16 stress-associated modules is provided as a heat map in Figure 3. Figures S5-S9. These figures are the same format as that of Figure 4, but represent cold (Figure S5), drought (Figure S6), salt (Figure S7), UV (Figure S8) and ozone stress (Figure S9). Each figure contains a heat map (top) and graphs of the expression of specific TEIncRNAs (bottom) that were chosen because they overrepresented with high interconnectivity. The heat graph shows transcript expression levels for genes and IncRNAs in each module (y-axis) and across conditions ( $x$-axis). The key to modules (yaxis) and stress conditions ( $x$-axis) are shown on the right legend, with conditions also separated by developmental stage (bottom of $x$-axis). Warmer colors within the heat map indicate high expression, and cooler colors are under-expression. The bar plots below the heat graph are eigenIncRNA expression values selected from the top overrepresented IncRNAs with high interconnectivity. The $x$-axis is the same as the heat map, and the id of the IncRNAs is provided by the color key.
\end{abstract}

Additional file 2: Table S1. A summary of pre-processing and alignment of Illumina and Pacbio datasets.

Additional file 3: Table S2. Details of identified IncRNAs including TE information.

Additional file 4: Table S3. Differentially expressed IncRNAs and TEIncRNAs under different abiotic stresses across developmental stages. Each sheet represents different stress treatments at a specific developmental stage. 
Additional file 5: Table S4. Information about stress-associated coexpression modules.

Additional file 6: Table S5. Details of stress-responsive modules, including member id, TE superfamily and TF family classification, membership value, kIM value and $p$-values across different stages and stress treatments.

Additional file 7: Table S6. Overrepresented GO terms associated with stress-responsive modules.

Additional file 8: Table S7. GO enrichment results for different stressresponsive modules. Each sheet includes enriched $\mathrm{GO}$ terms under difference abiotic stress such as heat, cold, drought, salt, UV and Ozone across V3 to V6 stages.

Additional file 9: Table S8. Blast results and sequence similarity of 39 maize hub IncRNAs compared to sorghum, rice and Arabidopsis.

Additional file 10: Table S9. A list of loci and primers used in GRT-PCR experiments.

\section{Abbreviations}

LncRNA: Long noncoding RNA; lincRNA: Long intergenic noncoding RNA; TE: Transposable element; TE-LncRNA: Transposon-derived LncRNA; GO: Gene ontology; FPKM: Fragments per kilobase per million mapped reads: LTR: Long terminal repeat; Pacbio: Pacific Biosciences; DEG: differentially expressed genes

\section{Acknowledgements}

The authors would like to acknowledge the support of High-Performance Computing Cluster at UC Irvine.

\section{Authors' contributions}

BSG and YDL conceived and designed the study. YDL and FQH performed the IncRNA discovery; YFZ and YDL performed the TE annotation. FQH and FLW performed the construction of co-expression network analysis, heat treatment and qRT-PCR experiment. FQH, YDL and BSG wrote the manuscript. All authors read and approved the manuscript.

\section{Funding}

This work was supported by financial support by National Natural Science Foundation of China (31771813), NSF grant DEB-1655808 to BSG, JAAS Exploratory and Disruptive Innovation Program (ZX (17)2015), National Natural Science Foundation of China (31601315) and Natural Science Foundation of Jiangsu Province (BK20160582). The funding body has no roles in in the design of the study and collection, analysis, and interpretation of data and in writing the manuscript.

\section{Availability of data and materials}

The datasets analysed during the current study are deposited in the publicly accessible NCBI Sequence Read Archive (SRA) Database and all accession numbers are available in the Additional file 6: Table S1.

\section{Ethics approval and consent to participate}

Not applicable.

\section{Consent for publication}

Not applicable.

\section{Competing interests}

The authors declare that they have no competing interests.

\section{Author details}

${ }^{1}$ Provincial Key Laboratory of Agrobiology, Institute of Crop Germplasm and Biotechnology, Jiangsu Academy of Agricultural Sciences, Nanjing, China. ${ }^{2}$ Department of Ecology and Evolutionary, Biology, UC Irvine, Irvine, CA, USA. ${ }^{3}$ Institute of Soil Science, Chinese Academy of Sciences, Nanjing, China. ${ }^{4}$ Department of Civil and Environmental, Engineering, UC Irvine, Irvine, CA, USA.
Received: 29 July 2019 Accepted: 30 October 2019

Published online: 15 November 2019

\section{References}

1. Mercer TR, Dinger ME, Mattick JS. Long non-coding RNAs: insights into functions. Nat Rev Genet. 2009;10:155-9.

2. Maeda N, Kasukawa T, Oyama R, Gough J, Frith M, Engström PG, et al. Transcript annotation in FANTOM3: mouse gene catalog based on physical cDNAs. PLoS Genet. 2006;2:e62.

3. Derrien T, Johnson R, Bussotti G, Tanzer A, Djebali S, Tilgner H, et al. The GENCODE v7 catalog of human long noncoding RNAs: analysis of their gene structure, evolution, and expression. Genome Res. 2012;22:1775-89.

4. Liu J, Jung C, Xu J, Wang H, Deng S, Bernad L, et al. Genome-wide analysis uncovers regulation of long Intergenic noncoding RNAs in Arabidopsis. Plant Cell. 2012;24:4333-45.

5. Zhang Y-C, Liao J-Y, Li Z-Y, Yu Y, Zhang J-P, Li Q-F, et al. Genome-wide screening and functional analysis identify a large number of long noncoding RNAs involved in the sexual reproduction of rice. Genome Biol. 2014; 15:512.

6. LV Y, Liang Z, Ge M, Qi W, Zhang T, Lin F, et al. Genome-wide identification and functional prediction of nitrogen-responsive intergenic and intronic long non-coding RNAs in maize (Zea mays L.). BMC Genomics. 2016;17:350.

7. Kapusta A, Kronenberg Z, Lynch VJ, Zhuo X, Ramsay L, Bourque G, et al. Transposable elements are major contributors to the origin, diversification, and regulation of vertebrate long noncoding RNAs. PLoS Genet. 2013;9: e1003470.

8. Li L, Eichten SR, Shimizu R, Petsch K, Yeh C-T, Wu W, et al. Genome-wide discovery and characterization of maize long non-coding RNAs. Genome Biol. 2014;15:R40.

9. Zhang H, Hu W, Hao J, Lv S, Wang C, Tong W, et al. Genome-wide identification and functional prediction of novel and fungi-responsive lincRNAs in Triticum aestivum. BMC Genomics. 2016;17:238.

10. Wang M, Yuan D, Tu L, Gao W, He Y, Hu H, et al. Long noncoding RNAs and their proposed functions in fibre development of cotton ( Gossypium spp .). 2015::1181-97.

11. Wang H, Chung PJ, Liu J, Jang IC, Kean MJ, Xu J, et al. Genome-wide identification of long noncoding natural antisense transcripts and their responses to light in Arabidopsis. Genome Res. 2014;24:444-53.

12. Golicz AA, Singh MB, Bhalla PL. The long intergenic noncoding RNA (lincRNA) landscape of the soybean genome. Plant Physiol. 2018;176:2133-47.

13. Yuan J, Li J, Yang Y, Tan C, Zhu Y, Hu L, et al. Stress-responsive regulation of long non-coding RNA polyadenylation in Oryza sativa. Plant J. 2018;93:814-27.

14. Cho J. Transposon-derived non-coding RNAs and their function in plants. Front Plant Sci. 2018;9:600.

15. Hadjiargyrou M, Delihas N. The intertwining of transposable elements and non-coding RNAs. Int J Mol Sci. 2013;14:13307-28.

16. Rinn JL, Chang HY. Genome regulation by long noncoding RNAs. Annu Rev Biochem. 2012;81:145-66.

17. Wang T, Zhao M, Zhang X, Liu M, Yang C, Chen Y, et al. Novel phosphate deficiency-responsive long non-coding RNAs in the legume model plant Medicago truncatula. J Exp Bot. 2017;68:5937-48.

18. Kim D-H, Sung S. Vernalization-triggered intragenic chromatin loop formation by long noncoding RNAs. Dev Cell. 2017;40:302-312.e4.

19. Seo JS, Sun H-X, Park BS, Huang C-H, Yeh S-D, Jung C, et al. ELF18-induced long-noncoding RNA associates with mediator to enhance expression of innate immune response genes in Arabidopsis. Plant Cell. 2017;29:1024-38.

20. Kopp F, Mendell JT. Functional classification and experimental dissection of long noncoding RNAs. Cell. 2018;172:393-407.

21. Kung JTY, Colognori D, Lee JT. Long noncoding RNAs: past, present, and future. Genetics. 2013;193:651-69.

22. Ben Amor B, Wirth S, Merchan F, Laporte P, d'Aubenton-Carafa Y, Hirsch J, et al. Novel long non-protein coding RNAs involved in Arabidopsis differentiation and stress responses. Genome Res. 2009;19:57-69.

23. Di C, Yuan J, Wu Y, Li J, Lin H, Hu L, et al. Characterization of stressresponsive IncRNAs in Arabidopsis thaliana by integrating expression, epigenetic and structural features. Plant J. 2014;80:848-61. https://doi.org/ 10.1111/tpj.12679.

24. Liu J, Jung C, Xu J, Wang H, Deng S, Bernad L, et al. Genome-wide analysis uncovers regulation of long intergenic noncoding RNAs in Arabidopsis. Plant Cell. 2012;24:4333-45. 
25. Zhu J-K. Abiotic stress signaling and responses in plants. Cell. 2016;167: 313-24.

26. Mittler R. Abiotic stress, the field environment and stress combination. Trends Plant Sci. 2006;11:15-9.

27. Masuka B, Araus JL, Das B, Sonder K, Cairns JE. Phenotyping for abiotic stress tolerance in maize. J Integr Plant Biol. 2012;54:238-49.

28. Gong F, Yang L, Tai F, Hu X, Wang W. Omics of maize stress response for sustainable food production: opportunities and challenges. Omics. 2014;18: 714-32.

29. Halford NG, Curtis TY, Chen Z, Huang J. Effects of abiotic stress and crop management on cereal grain composition: implications for food quality and safety. J Exp Bot. 2015;66:1145-56.

30. Zhang W, Han Z, Guo Q, Liu Y, Zheng Y, Wu F, et al. Identification of maize long non-coding RNAs responsive to drought stress. PLoS One. 2014;9: e98958.

31. Singh U, Khemka N, Rajkumar MS, Garg R, Jain M. PLnCPRO for prediction of long non-coding RNAs (IncRNAs) in plants and its application for discovery of abiotic stress-responsive IncRNAs in rice and chickpea. Nucleic Acids Res. 2017;45:e183.

32. Schnable PS, Ware D, Fulton RS, Stein JC, Wei F, Pasternak S, et al. The B73 maize genome: complexity, diversity, and dynamics. Science. 2009;326: 1112-5.

33. van Dam S, Võsa U, van der Graaf A, Franke L, de Magalhães JP. Gene coexpression analysis for functional classification and gene-disease predictions. Brief Bioinform. 2017;19:bbw139.

34. Signal B, Gloss BS, Dinger ME. Computational approaches for functional prediction and characterisation of long noncoding RNAs. Trends Genet. 2016:32:620-37.

35. Kelley D, Rinn J. Transposable elements reveal a stem cell-specific class of long noncoding RNAs. Genome Biol. 2012;13:R107.

36. Johnson R, Guigó R. The RIDL hypothesis: transposable elements as functional domains of long noncoding RNAs. RNA. 2014;20:959-76.

37. Tsoi LC, lyer MK, Stuart PE, Swindell WR, Gudjonsson JE, Tejasvi T, et al. Analysis of long non-coding RNAs highlights tissue-specific expression patterns and epigenetic profiles in normal and psoriatic skin. Genome Biol. 2015;16:24.

38. Wang G, Wang G, Zhong M, Wang J, Zhang J, Tang Y, et al. Genome-wide identification, splicing, and expression analysis of the myosin gene family in maize (Zea mays). J Exp Bot. 2014;65:923-38.

39. Amar D, Safer $H$, Shamir R. Dissection of regulatory networks that are altered in disease via differential co-expression. PLoS Comput Biol. 2013;9: e1002955.

40. Serin EAR, Nijveen $H$, Hilhorst HWM, Ligterink W. Learning from coexpression networks: possibilities and challenges. Front Plant Sci. 2016;7:444

41. Borrill P, Harrington SA, Simmonds J, Uauy C. Identification of transcription factors regulating senescence in wheat through gene regulatory network modelling. Plant Physiol. 2019::pp.00380.2019.

42. Varala K, Marshall-Colón A, Cirrone J, Brooks MD, Pasquino AV, Léran S, et al. Temporal transcriptional logic of dynamic regulatory networks underlying nitrogen signaling and use in plants. Proc Natl Acad Sci U S A. 2018;115:6494-9.

43. Mukhopadhyay P, Tyagi AK. OsTCP19 influences developmental and abiotic stress signaling by modulating ABI4-mediated pathways. Sci Rep. 2015;5:9998.

44. Chen L, Han J, Deng X, Tan S, Li L, Li L, et al. Expansion and stress responses of AP2/EREBP superfamily in Brachypodium Distachyon. Sci Rep. 2016;6:21623.

45. Vinocur B, Altman A. Recent advances in engineering plant tolerance to abiotic stress: achievements and limitations. Curr Opin Biotechnol. 2005; 16:123-32.

46. Umezawa T, Fujita M, Fujita Y, Yamaguchi-Shinozaki K, Shinozaki K. Engineering drought tolerance in plants: discovering and tailoring genes to unlock the future. Curr Opin Biotechnol. 2006;17:113-22.

47. Gaut BS. Evolutionary dynamics of grass genomes. New Phytol. 2002;154: $15-28$.

48. Gaut BS, Le Thierry d'Ennequin M, Peek AS, Sawkins MC. Maize as a model for the evolution of plant nuclear genomes. Proc Natl Acad Sci U S A. 2000; 97:7008-15.

49. Yang L, Duff MO, Graveley BR, Carmichael GG, Chen L-L. Genomewide characterization of non-polyadenylated RNAs. Genome Biol. 2011;12:R16.

50. Gaiti F, Fernandez-Valverde SL, Nakanishi N, Calcino AD, Yanai I, Tanurdzic $\mathrm{M}$, et al. Dynamic and widespread IncRNA expression in a sponge and the origin of animal complexity. Mol Biol Evol. 2015;32:2367-82.
51. Boerner S, McGinnis KM. Computational identification and functional predictions of long noncoding RNA in Zea mays. PLoS One. 2012;7:e43047.

52. Zhu Q-H, Wang M-B. Molecular functions of long non-coding rnas in plants. Genes (Basel). 2012;3:176-90

53. Baucom RS, Estill JC, Chaparro C, Upshaw N, Jogi A, Deragon J-M, et al. Exceptional diversity, non-random distribution, and rapid evolution of retroelements in the B73 maize genome. PLoS Genet. 2009;5:e1000732.

54. Bousios A, Gaut BS. Mechanistic and evolutionary questions about epigenetic conflicts between transposable elements and their plant hosts. Curr Opin Plant Biol. 2016;30:123-33.

55. Yan P, Luo S, Lu JY, Shen X. Cis- and trans-acting IncRNAs in pluripotency and reprogramming. Curr Opin Genet Dev. 2017;46:170-8.

56. Kornienko AE, Guenzl PM, Barlow DP, Pauler FM. Gene regulation by the act of long non-coding RNA transcription. BMC Biol. 2013;11:59.

57. Vandepoele K, Quimbaya M, Casneuf T, De Veylder L, Van de Peer Y. Unraveling transcriptional control in Arabidopsis using cis-regulatory elements and coexpression networks. Plant Physiol. 2009:150:535-46.

58. Jeong H, Mason SP, Barabási A-L, Oltvai ZN. Lethality and centrality in protein networks. Nature. 2001;411:41-2.

59. Barabási A-L, Oltvai ZN. Network biology: understanding the cell's functional organization. Nat Rev Genet. 2004:5:101-13.

60. Singh KB, Foley RC, Oñate-Sánchez L. Transcription factors in plant defense and stress responses. Curr Opin Plant Biol. 2002;5:430-6.

61. Agarwal PK, Jha B. Transcription factors in plants and ABA dependent and independent abiotic stress signalling. Biol Plant. 2010;54:201-12.

62. Lawlor D. Abiotic stress adaptation in plants. Physiological, molecular and genomic foundation. Ann Bot. 2011;107:vii.

63. Chuong EB, Elde NC, Feschotte $C$. Regulatory activities of transposable elements: from conflicts to benefits. Nat Rev Genet. 2017;18:71-86.

64. Trizzino M, Park Y, Holsbach-Beltrame M, Aracena K, Mika K, Caliskan M, et al. Transposable elements are the primary source of novelty in primate gene regulation. Genome Res. 2017;27:1623-33.

65. Chuong EB, Elde NC, Feschotte C. Regulatory evolution of innate immunity through co-option of endogenous retroviruses. Science. 2016;351:1083-7.

66. Wang B, Tseng E, Regulski M, Clark TA, Hon T, Jiao Y, et al. Unveiling the complexity of the maize transcriptome by single-molecule long-read sequencing. Nat Commun. 2016;7:11708.

67. Li P, Cao W, Fang H, Xu S, Yin S, Zhang Y, et al. Transcriptomic profiling of the maize (Zea mays L.) leaf response to abiotic stresses at the seedling stage. Front. Plant Sci. 2017:8:290.

68. Mimura M, Zallot R, Niehaus TD, Hasnain G, Gidda SK, Nguyen TND, et al. Arabidopsis $\mathrm{TH} 2$ encodes the orphan enzyme thiamin monophosphate phosphatase. Plant Cell. 2016;28:2683-96.

69. Makarevitch I, Waters AJ, West PT, Stitzer M, Hirsch CN, Ross-lbarra J, et al. Transposable elements contribute to activation of maize genes in response to abiotic stress. PLoS Genet. 2015;11:e1004915.

70. Cox MP, Peterson DA, Biggs PJ. SolexaQA: at-a-glance quality assessment of Illumina second-generation sequencing data. BMC Bioinformatics. 2010;11:485.

71. Dobin A, Davis CA, Schlesinger F, Drenkow J, Zaleski C, Jha S, et al. STAR: ultrafast universal RNA-seq aligner. Bioinformatics. 2013;29:15-21.

72. Pertea M, Pertea GM, Antonescu CM, Chang T-C, Mendell JT, Salzberg SL. StringTie enables improved reconstruction of a transcriptome from RNA-seq reads. Nat Biotechnol. 2015;33(3):290-5.

73. Li H. Minimap2: pairwise alignment for nucleotide sequences. Bioinformatics. 2018;34:3094-100

74. Wang D, Qu Z, Yang L, Zhang Q, Liu Z-H, Do T, et al. Transposable elements (TEs) contribute to stress-related long intergenic noncoding RNAs in plants. Plant J. 2017:90:133-46.

75. Sun L, Luo H, Bu D, Zhao G, Yu K, Zhang C, et al. Utilizing sequence intrinsic composition to classify protein-coding and long non-coding transcripts. Nucleic Acids Res. 2013;41:e166-6.

76. Johnson LS, Eddy SR, Portugaly E. Hidden Markov model speed heuristic and iterative HMM search procedure. BMC Bioinformatics. 2010;11:431.

77. Pertea M, Kim D, Pertea GM, Leek JT, Salzberg SL. Transcript-level expression analysis of RNA-seq experiments with HISAT. StringTie and Ballgown Nat Protoc. 2016;11:1650-67.

78. Li H, Handsaker B, Wysoker A, Fennell T, Ruan J, Homer N, et al. The sequence alignment/map format and SAMtools. Bioinformatics. 2009;25:2078-9.

79. Liao Y, Smyth GK, Shi W. featureCounts: an efficient general purpose program for assigning sequence reads to genomic features. Bioinformatics. 2014;30:923-30. 
80. Love Ml, Huber W, Anders S. Moderated estimation of fold change and dispersion for RNA-seq data with DESeq2. Genome Biol. 2014;15:550.

81. Langfelder P, Horvath S. WGCNA: an R package for weighted correlation network analysis. BMC Bioinformatics. 2008;9:559.

82. Bastian M, Heymann S, Jacomy M. Others. Gephi: an open source software for exploring and manipulating networks. Icwsm. 2009;8:361-2.

83. Apweiler R. UniProt: the universal protein knowledgebase. Nucleic Acids Res. 2004:32:115D-19.

84. Altschul S, Madden TL, Schäffer AA, Zhang J, Zhang Z, Miller W, et al. Gapped BLAST and PSI-BLAST: a new generation of protein database search programs. Nucleic Acids Res. 1997;25:3389-402.

85. Tian T, Liu Y, Yan H, You Q, Yi X, Du Z, et al. agriGO v2.0: a GO analysis toolkit for the agricultural community, 2017 update. Nucleic Acids Res. 2017; 45:W122-9.

86. Lalitha S. Primer Premier 5. Biotech Software \& Internet Report, 2000;1(6): 270-272.

\section{Publisher's Note}

Springer Nature remains neutral with regard to jurisdictional claims in published maps and institutional affiliations.

Ready to submit your research? Choose BMC and benefit from:

- fast, convenient online submission

- thorough peer review by experienced researchers in your field

- rapid publication on acceptance

- support for research data, including large and complex data types

- gold Open Access which fosters wider collaboration and increased citations

- maximum visibility for your research: over $100 \mathrm{M}$ website views per year

At BMC, research is always in progress.

Learn more biomedcentral.com/submissions 